\title{
CANCER PATIENTS' AWARENESS OF THEIR DISEASE AND PROGNOSIS
}

\author{
Mahmoud Al-Ahwal, MB, ChB, FRCPC
}

Doctors in different countries have various approaches to bioethical problems. Oncologists face ethical dilemmas during their daily practice with newly diagnosed cancer patients, particularly with regards to the disclosure of information about their diagnosis and prognosis. Medical literature suggests that most patients want to be told the truth about a diagnosis of cancer. ${ }^{1,2}$ In Saudi Arabia this issue is still not clear among physicians, patients and their families. Full disclosure of the presence of cancer to newly diagnosed patients is not well accepted by the majority of families. Most family members of cancer patients request that their patient should not be informed about the diagnosis of cancer and its prognosis. This attitude will, inevitably, influence the decision of a majority of doctors in giving cancer patients information about diagnosis, prognosis, treatment options and the side effects of treatment. This study has been conducted to provide some guidelines and draw some conclusions with regards to the issue of disclosure of information to patients diagnosed with cancer in our community.

\section{Material and Methods}

The study was conducted between January 1996 and March 1997. A questionnaire was distributed to three subject groups (cancer patients, doctors, and a group of normal patients) randomly selected from our population, regarding disclosure of information to cancer patients. Almost all the major hospitals dealing with cancer patients in the Western region of Saudi Arabia participated in this study. The ages of participants ranged from 20 to 60 years, with a median age of 45 years. The majority of subjects $(112 / 136$, or $82 \%)$ were Saudis. The questionnaire consisted of four main questions with three optional answers to each question. The questions were as follows: 1) What is your opinion of providing full information to a newly diagnosed cancer patient about his/her disease and prognosis?; 2) If you were diagnosed with cancer, would

From the Department of Medicine, King Abdulaziz University Hospital, Jeddah, Saudi Arabia.

Address reprint requests and correspondence to Dr. Al-Ahwal: Department of Medicine, King Abdulaziz University Hospital, P.O. Box 6615, Jeddah 21452, Saudi Arabia.

Accepted for publication 30 November 1997. Received 6 Augus 1997. you want to be fully informed about the diagnosis and prognosis?; 3) To whom should we provide the full information?; and for doctors only, 4) If the patient wanted to know details of his/her disease and prognosis, what would you do? The answers were collected and analyzed. Simple descriptive statistical analysis was performed, including mean, range and various frequency distributions.

\section{Results}

One hundred and thirty-six people participated in this study (33 cancer patients, 63 doctors, and 40 laypeople). Out of the 136 subjects, 112 were Saudis (31 patients, 41 doctors and 40 laypeople). Male to female ratio was 1:2 for cancer patients, 3:1 for doctors and 1:1 for laypeople. Their ages ranged between 20 and 60 years in both groups of doctors and laypeople. The patients were selected in the same range of age to match the other groups. Fifteen out of 40 laypeople were relatives of cancer patients undergoing treatment. Twenty-three doctors $(36.5 \%)$ were involved with cancer patients on a frequent basis, and 40 doctors $(63.5 \%)$ were involved with cancer patients only occasionally. Each group was analyzed separately, then added together to obtain a general opinion about the issue. In the cancer patients' group, 20 out of 33 (60.6\%) were in favor of informing the newly diagnosed cancer patient about his/her diagnosis and prognosis, while almost onethird $(30.3 \%)$ were not. In the second question, as to whether they themselves would want to know the truth if they had been diagnosed with cancer, 27 out of $33(81.7 \%)$ said they would like to be informed, while only $4(12.1 \%)$ said they would not. In the third question, 22 out of 33 patients $(66.7 \%)$ said they would like the patient and relatives to be informed about the diagnosis and prognosis, while $2(6.1 \%)$ said only the patient should be informed, and $8(24.2 \%)$ said only the relatives should be informed. From the doctor's group, 55 out of $63(87.3 \%)$ were in favor of providing full information to cancer patients, while only five doctors $(7.9 \%)$ were not. Regarding the second question, 58 doctors (92\%) said they would like to be informed if they had cancer, while only four $(6.3 \%)$ said they would prefer not to be informed. For the third question, 38 out of 63 doctors $(60 \%)$ said they would like the patient and his/her relatives to be informed about diagnosis and prognosis, as compared to eight $(12.7 \%)$ who wanted the patient alone to be given the information, and $17(27 \%)$ who wanted only the relatives to know. 
Regarding the fourth question, what a doctor would do if a cancer patient required full disclosure of his condition, 39 out of 63 doctors $(61.9 \%)$ said they would provide full information in a simplified way, and $24(38.1 \%)$ said they would not. For the third group (laypeople), 34 out of 40 $(85 \%)$ were in favor of full disclosure of facts to the newly diagnosed cancer patients about their disease, while only 4 $(10 \%)$ were not. If they themselves were diagnosed to have cancer, 37 out of $40(93 \%)$ said they would like to be informed while only $2(5 \%)$ said they would not. For the third question, 29 out of $40(72.5 \%)$ said they would like the patient and his relatives to be informed, compared to 2 $(5 \%)$ for patient alone and $9(22.5 \%)$ for relatives alone.

The results of all three groups together revealed that 109 out of $136(80.2 \%)$ are definitely in favor of full disclosure to newly diagnosed cancer patients, while 19 (14\%) were not. Total answers to the second question in favor of full disclosure were 121 out of $136(89.6 \%)$, while $10(7.4 \%)$ were not. Regarding the third question, 89 out of 136 answers $(65.4 \%)$ said they wanted full information to be given to the patient and his relatives together, 34 (25\%) to relatives alone and $12(8.8 \%)$ to the patient alone. These results indicated that all three groups are in favor of providing full information to cancer patients and their families regarding the diagnosis and prognosis.

\section{Discussion}

The most frequently discussed ethical issue within the context of cancer treatment is whether patients should be told their diagnosis. Doctors in different countries approach bioethical problems in various ways. The Saudi public exhibits a considerable degree of anxiety about cancer. This is either because of misinformation or poor information. ${ }^{3}$ Oncologists and other doctors dealing with cancer face ethical dilemmas in the course of their practice, in informing the newly diagnosed cancer patient about their diagnosis and prognosis. This issue of what to say and to whom, regarding the diagnosis of any serious illness such as cancer, is still not clear in our community. Until now the majority of our newly diagnosed cancer patients have been unaware of their diagnosis, a practice which is similar to that in many other countries, such as Italy ${ }^{4}$ and Taiwan. ${ }^{5}$

Our study indicates that $60.6 \%$ of our patients want to be told the truth about the diagnosis of cancer, which is similar to reports from many other countries. ${ }^{1,6}$ With regards to who should be provided this information, twothirds $(66.7 \%)$ of our patients want both the patient and his/her relatives to be informed. The reason behind this is the strong relationship between family members in our community. The rate of $24 \%$ of patients against full disclosure to the patient himself is similar to what was observed in a survey from the United Kingdom in $1954 .{ }^{7}$ In recent years, however, almost all patients in England have been well informed about their diagnosis, prognosis and treatment options, but their emotional well-being has rarely been probed. ${ }^{8}$ Doctors in our community are still unable to disclose full information to cancer patients about their disease. This is usually because of family wishes, poor availability of psychosocial resources to deal with the consequences of full disclosure, and the reluctance of Saudi patients to seek psychological support. Concealing the diagnosis from cancer patients may lead to poor patient compliance, misinformation of treatment options and side effects. This may have an impact on the patient's survival. Our study showed that the majority of doctors in our community $(87.3 \%)$, especially oncologists, are in favor of full disclosure to cancer patients, a rate which is similar to that in the majority of developed countries. Regarding who should be given the information, doctors and patients preferred that both patients and their relatives be informed together. This is because of the degree of social and psychological support the family offers to the patient. The wishes of the patient should also be respected regarding disclosure of information to the family. ${ }^{9}$

Deciding how and when to tell a patient that he/she has cancer can be a daunting task. If it is done with empathy and sensitivity, it can create an important bond between the doctor and patient. ${ }^{10}$ Our study shows that $61.9 \%$ of doctors prefer to give full information in a simplified way when the patient asks them. The optimal timing of full disclosure of information is before treatment. This will avoid the creation of barriers and lasting hostility.

In summary, this study revealed several areas where cancer patients are uninformed or misinformed about their diagnosis, prognosis and treatment options. This may have an impact on patient compliance with the treatment. In general, all three groups in our study were in favor of providing full information to cancer patients and their families about the diagnosis and prognosis in a simplified way. Health care professionals engage in sensitive communication and achieve a delicate balance between the patient's right to confidentiality and meeting the family members' need for information. Educational efforts towards public awareness that cancer is a treatable disease with a potential for cure have a major role in early diagnosis and cure. This, as well as psychosocial support, will relieve the anxiety of the public about the diagnosis, treatment of cancer and its consequences.

\section{References}

1. Asai A. Should physicians tell patients the truth? West J Med 1995;1:36-9.

2. Meredith C, Symonds P, Webster L, Lamont D, Pyper E, Gillis CR, et al. Information needs of cancer patients in West Scotland: crosssectional survey of patients' views. BMJ 1996;7059:724-6.

3. Bedikian AY, Thompson SE. Saudi community attitude towards cancer. Ann Saudi Med 1985;5:161-7.

4. Pronzato P, Bertelli G, Losardo P, et al. What do advanced cancer patients know of their disease? A report from Italy. Support Care Cancer 1994;4:242-4.

5. Ger LP, Ho ST, Chiang HH, et al. Cancer patients' knowledge of their diagnoses. J Formos Med Assoc 1996;8:605-11.

6. Hamajima N, Tajima K, Morishita M, et al. Patients' expectations of information provided at cancer hospitals in Japan. J Clin Oncol (Japan) 1996;5:362-7.

7. Paterson R, Aitken-Swan J. Public opinion on cancer: a survey among women in the Manchester area. Lancet 1954;2:857-61. 
8. Ford S, Fallowfield L, Lewis S. Doctor-patient interactions in oncology. Soc Sci Med 1996;11:1511-9.

9. Benson J, Britten N. Respecting the autonomy of cancer patients when talking with their families: qualitative analysis of semistructured interviews with patients. BMJ 1996;7059:729-31.

10. Bennett M, Alison D. Discussing the diagnosis and prognosis with cancer patients. Postgrad Med J 1996;843:25-9. 